\author{
Jolanta Sękowska*
}

\title{
WIE VIEL INFORMATION KÖNNEN WIR ANTIZIPIEREN? ZUM PROBLEM DER INKREMENTALITÄT UND UNEINGESCHRÄNKTEN INTERAKTIVITÄT BEIM SATZVERSTEHEN
}

\section{Einführung}

Der Begriff der Inkrementalität ergibt sich aus der intuitiven Idee, dass die Menschen die sprachlichen Äußerungen sofort interpretieren, ohne auf das Satzende oder Phrasengrenzen zu warten. Jede neu erscheinende lexikalische Einheit wird unmittelbar verarbeitet und interpretiert, noch bevor die nächste erscheint. Dies erfordert eine Eingliederung der kontinuierlich wahrgenommenen lexikalischen Einheiten in die sprachlich wohlgeformte und zumindest teilweise interpretierbare Satzrepräsentation, die auf der Basis des vorher auditiv oder visuell wahrgenommenen Inputs erstellt wurde. Selbst wenn es in der Fachliteratur eine lebhafte Debatte über die Informationstypen gibt, auf deren Grundlage die Entscheidungen zur Inkorporierung getroffen werden, worauf noch näher eingegangen wird, bildet in den letzten Jahrzehnten die Erkenntnis, dass die Sätze in einer höchst inkrementellen Weise interpretiert werden, den markantesten und vielleicht einzigen Punkt in der Satzverstehensforschung, in dem sich alle weitgehend einig sind. Unklar ist jedoch, inwieweit die Inkrementalität den effizienten Aufbau einer wohlgeformten und vollständigen Satzrepräsentation sicherstellen kann. Die legitime Frage nach den Grenzen der Inkrementalität wird im Folgenden detailliert behandelt.

${ }^{*}$ Dr. Jolanta Sękowska, Maria-Curie-Skłodowska-Universität in Lublin, Institut für Germanistik und Angewandte Linguistik, Pl. Marii Curie-Skłodowskiej 4a, 20-031 Lublin. E-Mail: jolanta. sekowska@poczta.umcs.lublin.pl 


\section{Problemaufriss}

Die Vorstellung allein, wonach das inkrementelle Satzverstehen darauf beruht, dass die Wörter sofort in einem links-nach-rechts-Modus analysiert werden, ist zu trivial. Vielmehr geht es bei der Inkrementalität darum, dass zu jedem Zeitpunkt des Satzverstehens eine möglichst komplexe Interpretation des Inputs durchgeführt wird. Die inkrementelle Satzverarbeitung als fundiertes Prinzip der prozessorientierten Psycholinguistik entspringt den grundlegenden Annahmen über die Architektur des Satzverstehenssystems, das schnell und effizient operieren soll, indem es eine maximale, geringstenfalls partielle Interpretation bereitstellt, sobald der Input empfangen wird, und zwar eine solche, die schnell in den vorliegenden Kontext integriert werden kann. Um diesen Effizienzanforderungen gerecht zu werden, sollen die Analyse und Interpretation der perzipierten lexikalischen Einheiten so schnell wie möglich voranschreiten. Andernfalls müssten sämtliche lexikalische Elemente zunächst unstrukturiert im Arbeitsgedächtnis abgelegt werden, was zu seiner Überbelastung führen könnte.

Dem inkrementellen Satzverstehen haftet jedoch ein Problem an, das sich aus der zeitlichen Dimension der Verarbeitungsprozesse ergibt und darin wurzelt, dass nicht zu jedem Zeitpunkt des Satzverstehens hinreichend Daten vorliegen, um eine sichere Entscheidung zur Analyse und Interpretation zu treffen. Entweder wird der Satz sofort hypothesengeleitet (top-down) analysiert und interpretiert, mit dem einkalkulierten Risiko, dass diese Analyse und Interpretation durch die später erscheinenden Daten nicht bestätigt werden, oder es wird gewartet, bis hinlänglich Daten vorhanden sind (bottom-up), was zwar eine sichere Interpretation durchführen lässt, aber die Gefahr mit sich bringt, dass mit der Satzinterpretation zu lange gewartet wird. Eine reine Bottom-up-Verarbeitung kann somit nicht als ein realer Mechanismus des inkrementellen Satzverstehens gelten, weil in kopffinalen Strukturen die Satzrepräsentation erst dann aufgebaut werden könnte, sobald das satzfinale Verb erkannt wird. Dies würde zu inplausiblen Verzögerungen beim Satzverstehen führen. Demgegenüber ist die Top-down-Analyse zwar stark inkrementell, weil die Satzrepräsentation erstellt wird, noch bevor einzelne Konstituenten sichtbar werden, was den Satz ziemlich schnell durch eine sofortige Integration der Inputwörter in die Struktur analysieren lässt, doch ist sie mit dem Risiko verbunden, dass bei komplexen zentralen Einbettungen den falschen strukturellen Hypothesen zu lange gefolgt wird. Es besteht somit eine Diskrepanz zwischen der Schnelligkeit und Zuverlässigkeit der Verarbeitung. Diese ist zumindest teilweise durch die Annahme einer Kombination der datenbasierten und hypothesengeleiteten Verfahren aufzulösen, indem bei jedem Analyseschritt zuerst die linke Ecke der Satzrepräsentation (left corner) erstellt wird ${ }^{1}$.

${ }^{1}$ Bezüglich einer formalen Beschreibung des Algorithmus vgl. Naumann, Langer (1994, S. $83 \mathrm{ff}$.) und Yoo (2007, S. 16ff.). 
Hier lässt schon das erste Inputwort eine hypothetische Fortsetzung konstruieren, deren erste Konstituente eben die linke Ecke ist, wodurch die noch ausstehenden Konstituenten so weit wie möglich vorausgesagt werden. Dadurch können später erscheinende lexikalische Einheiten schneller integriert werden, unter Umständen sogar direkt. Die Left-corner-Analyse erlaubt somit eine sofortige, inkrementelle Anbindung jedes Wortes an die Satzstruktur und damit seine unmittelbare Interpretation im Satzkontext. Gleichzeitig aber erfolgt kein Verarbeitungsschritt ohne Basis im Input, was Probleme der reinen Top-down- und Bottom-up-Analyse vermeiden lässt. Da jede hypothetische Expansion der Satzstruktur auf den Inputwörtern basiert, liegen die Vorteile des Left-corner-Verfahrens auf der Hand: Einerseits ist die Satzanalyse datenbasiert, andererseits wird hier auch eine prädiktive, antizipierende Komponente hinzugezogen, in dem Sinne, dass die Interpretation des Satzes nicht nur durch Inputeigenschaften, sondern auch durch Hypothesen zu möglichen Fortsetzungen des analysierten Satzteils vorangetrieben wird.

Belege für eine Kombination der datenbasierten und hypothesengeleiteten Satzverstehensprozesse liefern die sog. Garden-path-Strukturen. So kann man anhand des Satzes: „Ich glaube, dass Max zugunsten von Tim etwas unternommen wurde" exemplarisch zeigen, dass der Hörer/Leser nicht wartet, bis ausreichend Daten vorhanden sind, um bottom-up eine sichere Entscheidung zur Strukturanalyse zu treffen, sondern sich zu einer Analyse verpflichtet, die sich später mit weiteren Satzteilen unvereinbar erweist (vgl. dazu z. B. Bever 1970, S. 316; Hemforth, Konieczny 2008, S. 505ff.). Das obige Beispiel illustriert weiterhin, dass die prädiktive Top-down-Komponente auch bestimmten Einschränkungen unterliegt. Wären mehrere Hypothesen zu möglichen Fortsetzungen des Satzteils „Max“ aufgestellt, d.h. wäre man allen potentiellen Analysen - darunter auch der richtigen - von Anfang an gefolgt, dann sollte es zu keinem Garden-path-Effekt gekommen sein. Die Frage ist nun, wodurch die Hypothesenbildung und prädiktive Verarbeitung eingeschränkt werden. Anders gesagt: Wo liegen die Grenzen unserer Antizipationsmöglichkeiten beim Satzverstehen?

\section{Antizipationsrahmen in modularen und interaktiven Modellen des Satzverstehens}

Die modularen und interaktiven Modelle des Satzverstehens unterscheiden sich in Bezug auf den Antizipationsrahmen. In den modularen Modellen wird die Antizipation syntaktisch eingeschränkt, indem angenommen wird, dass die initiale Satzanalyse ausschließlich auf der Grundlage der syntaktischen Prinzipien durchgeführt wird (vgl. Frazier 1990, S. 409ff.; Gorrell 1995, S. 149ff.; Friederici 2002, S. $78 \mathrm{ff}$;; Friederici, Kotz 2003, S. S8ff.). Jede neu erscheinende lexikalische Einheit 
wird in die top-down erstellte syntaktische Struktur auf eine solche Art und Weise inkorporiert, dass zugleich eine Expansion der Phrasenstruktur vorausgesagt wird. Die prädiktive Komponente bezieht sich somit auf die Syntax. So beginnt die Analyse mit der Erstellung einer grundlegenden SO-Satzstruktur, innerhalb derer die Inputelemente verschiedene strukturelle Positionen von links nach rechts besetzen. Ein solches Verfahren ist aus der Effizienzperspektive von Vorteil: Da initial nur eine syntaktische Analyse eingeschlagen wird, ist das Satzverstehen mit einem begrenzten kognitiven Aufwand verbunden ${ }^{2}$. Im zweiten Verarbeitungsschritt wird überprüft, ob die initial eingeschlagene Analyse mit Informationen aus anderen Wissensquellen wie Belebtheit, semantische Plausibilität, Kontext und Frequenz kompatibel ist. Falls dies nicht zutrifft, wie in dem oben angegebenen Beispiel, kommt es zu ihrer Revision (Reanalyse). Zwar ist die Reanalyse mit einem gewissen kognitiven Aufwand verbunden, aber das Satzverstehenssystem geht das Risiko ein, dass sich die initiale Analyse als falsch herausstellen kann.

Eine ganz andere Vorgehensweise wird in den interaktiven/constraint-basierten Modellen des Satzverstehens postuliert, deren Entstehung durch die fortschreitende Lexikalisierung der linguistischen Theorien vorangetrieben wurde (vgl. z. B. MacDonald, Pearlmutter, Seidenberg 1994, S. 676ff.; Trueswell, Tanenhaus 1994, S. 155ff.; Trueswell, Tanenhaus, Garnsey 1994, S. 304f.; McRae, Spivey-Knowlton, Tanenhaus 1998, S. 305ff.; Vosse, Kempen 2000, S. 136ff.) ${ }^{3}$. Hier wird von einem holistisch organisierten Wissen ausgegangen, in dem Sinne, dass das Lexikon, das eine repräsentationale Wissensbasis bildet, sowohl Informationen über potentielle syntaktische Strukturen, darunter auch Argumentstrukturen, für die jeweilige lexikalische Einheit als auch Informationen über ihre semantische Plausibilität und Frequenz beinhaltet. Zu jedem Zeitpunkt des Satzverstehens wird Zugang zu allen sprachlichen und nicht-sprachlichen Wissensdomänen gewährleistet. Alternative Satzinterpretationen werden parallel erstellt und für diesen Zweck werden alle zugänglichen Wissensquellen herangezogen. Die Satzverstehensprozesse operieren hier auf einer einheitlichen Repräsentationsebene und sind somit weder domänenspezifisch noch informationell verkapselt. Alle Informationstypen werden auch gleichzeitig in den frühesten Stadien des Satzverstehens berücksichtigt und der Aufbau der syntaktischen Struktur wird durch alle zugänglichen Wissensquellen determiniert, auch durch kontextuelles Wissen, Informationen über die Vorkommenshäufigkeit der jeweiligen Struktur (Frequenz) und pragmatisches Wissen.

${ }^{2}$ Damit können auch Wortstellungspräferenzen, insbesondere die Subjekterstpräferenz, erklärt werden.

${ }^{3}$ Die Lexikalisierungstendenzen zeichneten sich sowohl in der Generativen Grammatik ab, wo auf die die syntaktische Struktur generierenden Phrasenstrukturregeln (Chomsky 1965) zugunsten der direkt mit den lexikalischen Einheiten assoziierten X-bar-Strukturen (Chomsky 1981) verzichtet wurde, als auch in der lexikalisch-funktionalen Grammatik (Bresnan 2001) und vor allem in der Konstruktionsgrammatik (Fillmore 1988; Fillmore, Kay, O'Connor 1988; Goldberg 1995). 
In den interaktiven/constraint-basierten Modellen des Satzverstehens wird mit dem ersten perzipierten Wort Zugang zu einem breiten Spektrum der sprachlichen und nicht-sprachlichen Informationen gewährt und die Interpretation des Satzes profitiert von Anfang an von entsprechendem Hintergrundwissen und Informationen über den Diskurskontext. Der Verarbeitungsstrom verläuft von lexikalischen Einheiten ausgehend ungehindert in alle Richtungen, d. h. die Verarbeitung des syntaktischen Wissens ist nur ein Teilbereich der Verarbeitung vieler anderer Wissensbestände, darunter semantischen und pragmatischen Wissens. Es gibt hier keine separaten lexikalischen und syntaktischen Verarbeitungsprozesse, weil alle Informationen, die in den lexikalischen Einheiten enthalten sind, samt dem Hintergrundwissen die Satzanalyse bestimmen und leiten. Daraus ergibt sich die „maximale“ Inkrementalität des Satzverstehens, wobei „maximal" sich auf die prädiktive Komponente bezieht: Bei einer Ambiguität werden mehrere Interpretationen parallel erstellt, die nicht nur auf dem syntaktischen oder morphosyntaktischen Wissen basieren, sondern auch alle möglichen Fortsetzungen des gerade analysierten Satzteils umfassen (vgl. z. B. Altman, Mirkovic 2009, S. 604) $)^{4}$.

Die Antizipation weist jedoch sowohl Vorteile als auch Nachteile auf. Als Vorteil kann die Fähigkeit des kognitiven Systems gelten, sich auf die Zukunft vorzubereiten. Als Nachteil ist zu werten, dass eine breite Antizipation, die über den Rahmen der Phrase oder noch mehr über den Satzrahmen hinausgeht, mit einem großen kognitiven Aufwand verbunden ist, insbesondere zu Beginn des Verarbeitungsprozesses. Es muss eine große Menge an Wissen im aktiven Zustand behalten werden. Erst im Laufe der Verarbeitung, wenn die Anzahl der Kontexte schrittweise eingeschränkt wird, kann der übermäßige Anstieg des Verarbeitungsaufwands verringert werden.

Distinkte Vorstellungen über die Zugänglichkeit der einzelnen Wissensdomänen in modularen und interaktiven Modellen des Satzverstehens wirken sich aber nicht nur qualitativ, sondern auch quantitativ auf die grundlegenden Annahmen zur Antizipation aus. So ist der initiale Antizipationsrahmen in modularen Modellen des Satzverstehens eng, weil das Top-down-Verfahren ausschließlich die syntaktische Struktur betrifft und die ersten Entscheidungen zur Satzanalyse auf der Grundlage des syntaktischen Wissens getroffen werden. Er breitet sich erst mit der Zeit aus, wenn andere Wissensquellen hinzugezogen werden (Abb. 1).

${ }^{4}$ Die Kontroverse zwischen den modularen und interaktiven Modellen des Satzverstehens betrifft somit den Zeitpunkt, an dem die semantischen oder konzeptuellen Informationen genutzt werden. Während die semantischen und konzeptuellen Informationen in modularen Modellen in einer späteren Verarbeitungsphase wirksam sind, werden in interaktiven Modellen die frühesten Stadien des Satzanalyseprozesses durch ein breites Spektrum an Informationen - darunter auch durch semantisches und konzeptuelles Wissen - beeinflusst. 


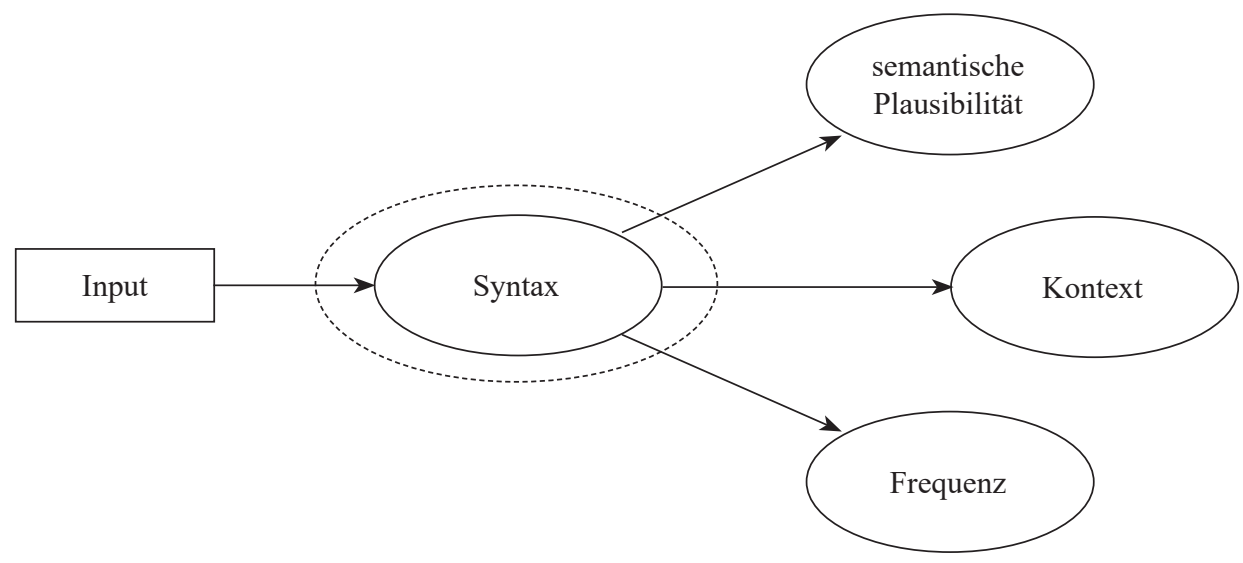

Abb. 1. Der initiale Antizipationsrahmen in modularen Modellen des Satzverstehens

In den interaktiven/constraint-basierten Modellen ist der initiale Antizipationsrahmen viel größer, weil schon beim ersten perzipierten Wort alle Wissensquellen (darunter sprachliches und nicht-sprachliches Wissen) aktiviert werden. Dieser wird schrittweise mit jeder perzipierten lexikalischen Einheit reduziert (Abb. 2). Die Kontroverse zwischen den modularen und interaktiven Modellen des Satzverstehens betrifft somit sowohl die Frage, was, als auch die, zu welchem Zeitpunkt antizipiert wird.

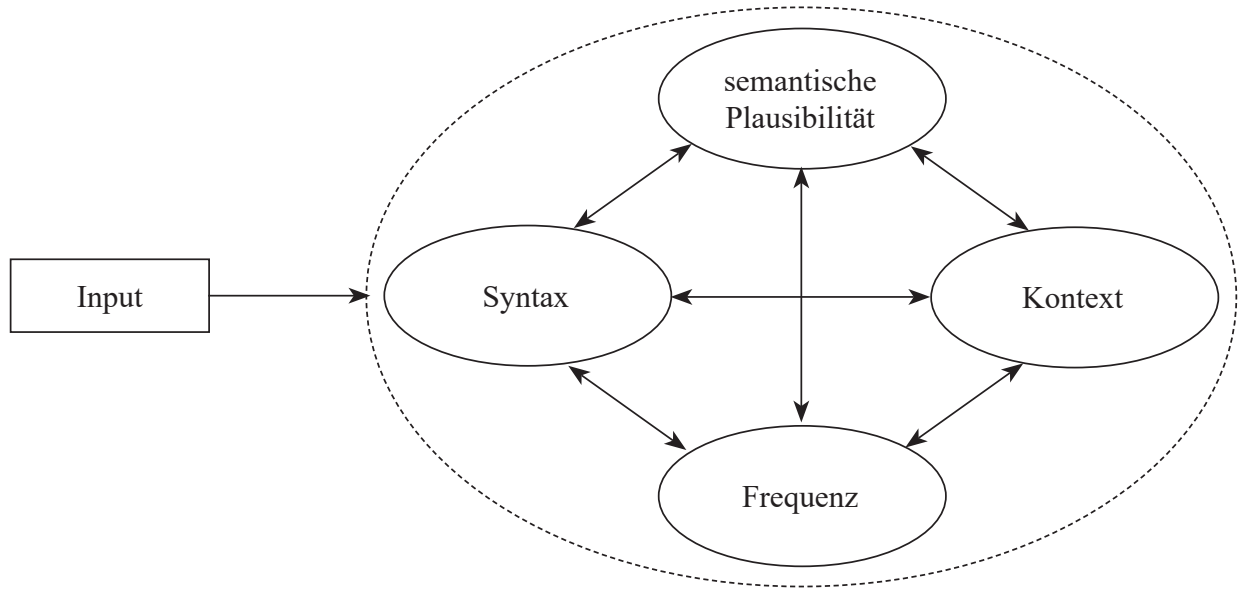

Abb. 2. Der initiale Antizipationsrahmen in modularen Modellen des Satzverstehens

Die erhöhte Lexikalisierung der repräsentationalen Wissensbasis wirft allerdings interessante Fragen über die Rolle der einzelnen Constraints beim inkrementellen Satzverstehen auf. Ein großer Antizipationsrahmen und - was damit 
zusammenhängt - starke Inkrementalität verleihen den interaktiven Modellen zumindest einen Vorteil: die Annahme einer Semantik, die für partielle Strukturen angewendet werden kann, was die Interpretation der Satzfragmente erlaubt, die in der Alltagssprache sehr oft vorkommen. Auf der anderen Seite weist der breite Antizipationsrahmen die Grenzen der Inkrementalität auf, weil mehrere, möglicherweise Dutzende von Regeln eine bestimmte Kategorie erweitern können, zwischen denen gewählt wird. Dann kann der Algorithmus mehrfach scheitern, bevor die richtige Vermutung aufgestellt wird. Damit hängt das knifflige Problem der interaktionistischen Zugangsweise zusammen, das darauf beruht, dass bei einem breiten Antizipationsrahmen der Input einfach nicht genug benutzt wird, um richtige Entscheidungen zur Satzinterpretation zu treffen.

\section{Schlussfolgerungen}

Die Annahme eines breiten Antizipationsrahmens und einer maximalen Inkrementalität ist aus den schon diskutierten Gründen der überdimensionalen kognitiven Belastung in den ersten Phasen des Satzverstehens nicht aufrechtzuerhalten. So ist es eine Herausforderung für die interaktiven Modelle des Satzverstehens, die prädiktiven Prozesse so einzuschränken, dass die potentiellen Strukturen nicht übergeneriert werden. Als eine Einschränkung gilt hier die Gewichtung der parallel verfolgten Analysen (z. B. Gibson 1991, S. 16ff.), was zwar Probleme der uneingeschränkten Antizipation, d. h. eine übermäßige Belastung des Arbeitsspeichers zu Beginn des Verarbeitungsprozesses umgehen und Garden-path-Effekte erklären lässt, aber zugleich die interaktiven Modelle dazu verpflichtet, klare Kriterien dafür anzugeben, auf welcher Grundlage die Gewichtung der Analysen erfolgt. Die Frage, ob die Frequenzdaten als ein solches Kriterium fungieren können - wie dies z. B. Jurafsky (2003, S. 39ff.) postuliert hat - bedarf einer empirischen Klärung, die umso schwieriger ist, als der Gebrauch von Frequenzdaten als Evidenzquelle äußerst problematisch erscheint und es fraglich ist, ob die Korpusdaten direkt auf die Satzverstehensprozesse übertragen werden können (vgl. dazu Featherston 2005, S. 187ff.). Die Erklärung eines Phänomens durch die Frequenz seines Auftretens setzt die Beantwortung der Frage voraus, was die Ursache der jeweiligen Frequenzverteilung ist. Nach Bornkessel-Schlesewsky, Bornkessel (2009, S. 101f.) ist nicht eindeutig, auf welcher Grundlage exakte frequenzbasierte Vorhersagen abgeleitet werden sollen. Laut Kempen, Harbusch (2004, S. 330ff.) und Crocker, Keller (2006, S. 239) werden nicht alle Wortstellungspräferenzen durch Unterschiede in der Korpusfrequenz widergespiegelt. ${ }^{5}$ So haben beispielsweise experimentelle Studien eine Präferenz für hohe Anbindung

\footnotetext{
5 Kempen, Harbusch (2004, S. 330) nennen dies frequency-grammaticality discrepancy.
} 
gezeigt, d. h. für die Anbindung einer PP an das Verb (vgl. z. B. Rayner, Carlson, Frazier 1983, S. 358ff.; Ferreira, Clifton 1986, S. 348ff.), während die Korpusanalysen aufdeckten, dass eine niedrige Anbindung, d.h. die Anbindung an die NP, ungefähr zweimal so häufig wie die Anbindung an das Verb auftritt (Hindle, Rooth 1993, S. 103ff.).

Auch die Ergebnisse der Studien zur Verarbeitung der sog. NP-S-Ambiguitäten sind alles andere als eindeutig. So haben Trueswell, Tanenhaus, Kello (1993, S. 528ff.) für die Sätze The student forgot the solution und The student forgot the solution was incorrect, in denen die Nominalphrase the solution in Bezug auf ihre syntaktische Funktion temporär ambig ist und entweder als direktes Objekt des Matrixsatzes oder Subjekt des Komplementsatzes fungieren kann, berichtet, dass die Präferenz in der Lesart frequenzbasiert ist, genauer gesagt, davon abhängt, wie oft das Verb des Matrixsatzes mit dem direkten Objekt oder Komplementsatz auftritt. Eine dazu konträre Datenlage haben jedoch Pickering, Traxler, Crocker (2000, S. 447ff.) erbracht, indem sie eine Präferenz für direktes Objekt auch für die Verben gezeigt haben, bei denen die intransitive Konstruktion dominiert. Eine Präferenz für die Anbindung einer ambigen NP an das Verb gegenüber einer Lesart als Adjunkt haben auch andere Studien nachgewiesen (Boland, Bohem-Jeringan, 1998, S. 684ff.; Schütze, Gibson, 1999, S. 409ff.). Weiterhin haben die Untersuchungen zur Verarbeitung der Verbumgebung Evidenz dafür geliefert, dass in kopffinalen Sprachen die Nominalphrasen vor dem subkategorisierenden Verb identifiziert und interpretiert werden (vgl. z. B. Bader, Lasser 1994, S. 225ff.; Kamide, Mit-

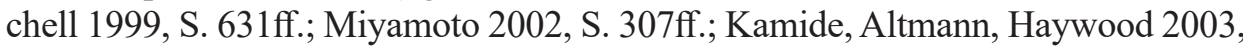
S. 133ff.; Aoshima, Phillips, Weinberg 2004, S. 23ff.; Pablos 2011, S. 3ff.), was als Beleg dafür gilt, dass die Anbindungspräferenzen nicht lexikalisch basiert sind. Eine mögliche Erklärung für dieses recht heterogene Bild, das sich aus dem heutigen Forschungsstand ergibt, können Untersuchungen zur Verarbeitung der obligatorischen und fakultativen Information, insbesondere der Verbbegleiter liefern. Die Hinweise, dass obligatorische Information fundamental anders als fakultative verarbeitet wird, sind zwar nicht spärlich gesät, aber im Gegensatz zur Verarbeitung obligatorischer Argumente des Verbs, für die robuste Präferenzen festgestellt werden konnten, sind die Ergebnisse der Studien für fakultative Ergänzungen stark variabel. Der Grund dafür kann darin liegen, dass der Status der Begleiter des Verbs im Hinblick auf ihre Prädiktionsmöglichkeiten wegen der Unzulänglichkeit ihrer bisherigen Klassifizierung in den experimentellen Bedingungen nicht adäquat bestimmt wurde. Um der Sache die richtigen Konturen zu verleihen, muss nämlich entschieden werden, ob die Verbbegleiter auf die Handlung verweisen oder nicht, d. h. ob auf ihrer Grundlage die Anzahl der Hypothesen zum satzfinalen Verb reduziert werden kann. Durch den falsch eingeschätzten Status der Satzglieder kann nämlich verschleiert werden, was und zu welchem Zeitpunkt antizipiert wird, weil nicht nur auf der Grundlage der obligatorischen, sondern auch fakultativen Information je nach Grad ihrer Beteiligung an der Verbhandlung und insbesondere je nachdem, ob die Information 
redundant ist oder nicht, Vorhersagen zur Fortsetzung des analysierten Satzteils gemacht werden können ${ }^{6}$.

Unabhängig davon, welche Einschränkungen für die Einbeziehung der Wissensquellen beim Satzverstehen vorliegen, entsteht das Endprodukt der Satzverstehensprozesse aus dem Zusammenspiel der syntaktischen, semantischen, und probabilistischen Informationen. Die Frage ist nun, inwieweit der Strukturaufbau lexikalisch getrieben ist, vorausgesetzt, dass das Satzverstehen inkrementell ist. Lombardo, Sturt (2002, S. 152) deuten beispielsweise darauf hin, dass die vollständig lexikalisch projizierten syntaktischen Strukturen eine inkrementelle Integration einer großen Anzahl von Wörtern ermöglichen, aber die Anbindung in vielen Fällen etwas mehr als eine lexikalisch basierte Struktur erfordert. Falls es zutrifft, dass nicht jede Integration im Rahmen eines vollständig lexikalisierten Modells erklärt werden kann, so wird die modulare Konzeption untermauert. Ansonsten spricht für die modulare Auffassung, dass es für das Satzverstehenssystem effizienter sein könnte, nur eine Analyse einzuschlagen, gesetzt den Fall, dass sie sich als falsch herausstellt, statt allen parallel erstellten Interpretationen - auch wenn sie rangiert sind - zu folgen. Auf der anderen Seite können die interaktiven/ constraint-basierten Modelle eine elegante Erklärung dafür liefern, wie sich die Präferenzen für bestimmte Lesarten im Laufe der Satzverarbeitung ändern. Hierzu besteht jedoch ein empirischer Klärungsbedarf, welche lexikalischen Informationen als Grundlage für die prädiktive Verarbeitung gelten können. Um ihn zu decken sind weitere Untersuchungen zur Rolle der fakultativen und obligatorischen Information beim Satzverstehen vonnöten.

\section{LITERATURVERZEICHNIS}

Altmann G.T.M., Mirković J. (2009), Incrementality and prediction in human sentence processing. In: Cognitive Science, H. 33, S. 583-609.

Aoshima S., Phillips C., Weinberg A. (2004), Processing filler-gap dependencies in a headfinal language. In: Journal of Memory and Language, H. 51, S. 23-54.

Bader M., Lasser I. (1994), German verb-final clauses and sentence processing: Evidence for immediate attachment. In: Clifton Ch./Frazier L./Rayner K. (Hgg.), Perspectives on Sentence Processing, Hillsdale NJ, S. 225-242.

Bever T.G. (1970), The cognitive basis for linguistic structures. In: Hayes J.R. (Hg.), Cognition and the development of language, New York, S. 279-362.

Boland J.E., Boehm-Jernigan H. (1998), Lexical constraints and prepositional phrase attachment. In: Journal of Memory and Language, H. 39, S. 684-719.

${ }^{6}$ Zur Problematik der Gliederung der syntaktischen Verbumgebung siehe die innovative Konzeption von Schwenk (2017a, S. 55ff., 2017b, S. 10ff.), nach der die Begleiter der deutschen Verben nach semantischen Kriterien auf vier Gruppen verteilt werden können, nämlich: (1) Mitgaben (= Komplemente), die durch die Tätigkeit impliziert sind; (2) Beigaben (= Adplemente), die in der Tätigkeit inkludiert (beinhaltet) sind; (3) Zugaben (= Supplemente), die in die Tätigkeit involviert sind; und (4) Angaben, also traditionelle adjunktive Elemente. 
Bornkessel-Schlesewsky I., Schlesewsky M. (2009), Processing Syntax and Morphology: A Neurocognitive Perspective, Oxford.

Bresnan J. (2001) (Hg.), Lexical-Functional Syntax, Oxford.

Chomsky N. (1965), Aspects of the Theory of Syntax, Cambridge.

Chomsky N. (1981), Lectures on Government and Binding. The Pisa Lectures, Dordrecht.

Crocker M.W., Keller F. (2006), Probabilistic grammars as models of gradience in language processing. In: Fanselow G./Féry C./Vogel R./Schlesewsky M. (Hgg.), Gradience in Grammar: Generative Perspectives, Oxford, S. 227-245.

Featherston S. (2005), The decathlon model of empirical syntax. In: Kepser S./Reis M. (Hgg.), Linguistic evidence - Empirical, theoretical, and computational perspectives, Berlin, S. 187-208.

Ferreira F., Clifton Ch. (1986), The independence of syntactic processing. In: Journal of Memory and Language, Bd. 25, H. 3, S. 348-368.

Fillmore Ch. (1988), The mechanisms of "Construction Grammar". In: Proceedings of the annual meeting of the Berkeley Linguistics Society, Bd. 14, S. 33-35.

Fillmore Ch., Kay P., O'Connor M.C. (1988), Regularity and idiomacity in grammatical constructions. In: Language, Bd. 64, H. 3, S. 501-538.

Frazier L. (1990), Exploring the architecture of language processing system. In: Altmann G.T.M. (Hg.), Cognitive Models of Speech Processing. Psycholinguistic and Computational Perspectives. Cambridge, S. 409-433.

Friederici A.D. (2002), Towards a neural basis of auditory sentence processing. In: Trends in Cognitive Sciences, Bd. 6, H. 2, S. 78-84.

Friederici A.D., Kotz S.A. (2003), The brain basis of syntactic processes: functional imaging and lesion studies. In: NeuroImage, H. 20, S. S8-S17.

Gibson E. (1991), A computational theory of linguistic processing: Memory limitations and processing breakdown, Dissertation, Carnegie Mellon University.

Goldberg A.E. (1995), Constructions. A Construction Grammar Approach to Argument Structure, Chicago.

Gorrell P. (1995), Syntax and parsing. Cambridge u.a.

Hemforth B., Konieczny L. (2008), Sätze verstehen und produzieren. In: Müsseler J./Prinz W. (Hgg.), Lehrbuch Allgemeine Psychologie, Heidelberg, S. 505-554.

Hindle D., Rooth, M. (1993), Structural Ambiguity and Lexical Relations. In: Computational Linguistics, H. 19, S. 103-120.

Jurafsky D. (2003), Probabilistic modelling in psycholinguistics: Linguistic comprehension and production. In: Bod R./Hay J./Jannedy S. (Hgg.), Probablistic Linguistics, Cambridge, MA, S. 39-96.

Kamide Y., Altmann G.T.M., Haywood S.L. (2003), The time-course of prediction in incremental sentence processing: Evidence from anticipatory eye movements. In: Journal of Memory and Language, H. 49, S. 133-156.

Kamide Y., Mitchell D.C. (1999), Incremental pre-head attachment in Japanese parsing. In: Language and Cognitive Processes, H. 14, S. 631-662.

Kempen G., Harbusch K. (2005), The relationship between grammaticality ratings and corpus frequencies: A case study into word order variability in the midfield of German clauses. In: Kepser S./Reis M. (Hgg.), Linguistic evidence - Empirical, theoretical, and computational perspectives, Berlin, S. 329-349.

Lombardo V., Sturt P. (2002), Incrementality and lexicalism: A treebank study. In: Merlo P./Stevenson S. (Hgg.), The Lexical Basis of Sentence Processing. Formal, computational and experimental issues. Amsterdam, Philadelphia, S. 137-156.

MacDonald M.C., Pearlmutter N.J., Seidenberg M.S. (1994), The lexical nature of syntactic ambiguity resolution. In: Psychological Review, Bd. 101, H. 4, S. 676-703. 
McRae K., Spivey-Knowlton M.J., Tanenhaus M.K. (1998), Modeling the Influence of Thematic Fit (and Other Constraints) in On-line Sentence Comprehension. In: Journal of Memory and Language, Bd. 38, H. 3, S. 283-312.

Miyamoto E.T. (2002), Case markers as clause boundary inducers in Japanese. In: Journal of Psycholinguistic Research, Bd. 31, H. 4, S. 307-347.

Naumann S., Langer H. (1994), Parsing - Eine Einführung in die maschinelle Analyse natürlicher Sprache, Stuttgart.

Pablos L. (2011), Rich agreement in Basque: Evidence for pre-verbal structure building. In: Yamashita H./Hirose Y./Packard J.L. (Hgg.), Processing and Producing Head-final Structures, Dordrecht u.a., S. 3-22.

Pickering M.J., Traxler M., Crocker M.W. (2000), Ambiguity resolution in sentence processing: Evidence against frequency-based accounts. In: Journal of Memory and Language, H. 43, S. 447-475.

Rayner K., Carlson M., Frazier L. (1983), The interaction of syntax and semantics during sentence processing: eye movements in the analysis of semantically biased sentences. In: Journal of Verbal Learning and Verbal Behavior, Bd. 22, H. 3, S. 358-374.

Schwenk H.-J. (2017a), Ergänzungen und Angaben und sonst nichts? Die syntaktische Umgebung des deutschen Verbs und ihre Gliederung, Frankfurt a. M. u.a.

Schwenk H.-J. (2017b), Exemplarisches Valenz- und Konstruktionswörterbuch deutscher Verben. Die Differenzierung und Klassifizierung der Begleiter des deutschen Verbs und ihre lexikographische Umsetzung in neuer Konzeption, Frankfurt a. M. u.a.

Schütze C.T., Gibson E. (1999), Argumenthood and English prepositional phrase attachment. In: Journal of Memory and Language, H. 40, S. 409-431.

Trueswell J.C., Tanenhaus M.K. (1994), Toward a lexicalist framework for constraint-based syntactic ambiguity resolution. In: Clifton C. Jr./Frazier L./Rayner K. (Hgg.), Perspectives in sentence processing, Hillsdale, NJ, S. 155-179.

Trueswell J.C., Tanenhaus, M.K., Garnsey S.M. (1994), Semantic influences on parsing: Use of thematic role information in syntactic ambiguity resolution. In: Journal of Memory and Language, Bd. 33, H. 3, S. 285-318.

Trueswell J.C., Tanenhaus M.K., Kello, C. (1993), Verb-specific constraints in sentence processing: Separating effects of lexical preference from garden-paths. In: Journal of Experimental Psychology: Learning, Memory and Cognition, H. 19, S. 528-553.

Vosse T., Kempen G. (2000), Syntactic structure assembly in human parsing: a computational model based on competitive inhibition and a lexicalist grammar. In: Cognition, Bd. 72, H. 2, S. 105-143.

Yoo D.G. (2007), Syntax und Kontext: Satzverarbeitung in kopffinalen Sprachen, Dissertation, Universität Bielefeld.

Jolanta Sękowska

\section{HOW MUCH DATA CAN WE ANTICIPATE? INCREMENTALITY AND UNLIMITED INTERACTIVITY IN SENTENCE COMPREHENSION}

(Summary)

This paper discusses the issue of the boundaries of incrementality in sentence-comprehension processes. The maximum incrementality, postulated as unlimited interactive sentence-comprehension models, allows anticipation and prediction top-down processing, facilitating quick interpretation 
of the perceived linguistic input. However, it involves a substantial strain on cognitive resources, especially at the beginning of the sentence. In addition, in the case of structures with verbs placed at the end of a sentence, unlimited interactivity keeps vast knowledge resources active until the verb and its arguments are agreed. This imposes a certain order of importance on interactive models at the same time assigned to the ongoing top-down processing, thus limiting incrementality, interactivity and the ability to anticipate.

Keywords: sentence comprehension, sentence processing, incrementality, anticipation, interactivity 